Result Bonemarrow tranplantation is the only curable treatment of this disease. About 3 million to 10 million taka need for this treatment. Above this situation we learn that prevention is only way to reduce this diseases. Screening system NESTROFT is available in our center. Only 10 taka need. Some cases DNA analysis will be need.

Conclusion Antenatal diagnosis is important and available in our center. If the foetus suffering from this disease legal termination of pregnancy should be need. This way we can free from the disease.

\section{HEMOLYTIC ANEMIA ASSOCIATED WITH INTRAVENOUS IMMUNOGLOBULIN}

doi:10.1136/archdischild-2012-302724.0518

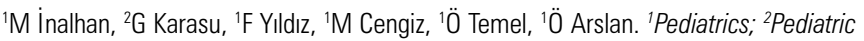
Hematology, Zeynep Kamil Maternity and Children Diseases Training and Research State Hospital, Istanbul, Turkey

Introduction Intravenous immunoglobulin (IVIG) associated hemolytic anemia is a potentially serious complication that is often overlooked. Here we describe a case of Kawasaki disease (KD) who recurrently developed coombs positive hemolytic anemia following IVIG administrations.

Case Report A three-years-old girl admitted with the complaint of fever, swelling of the hands and feet with palmar erythema. Investigations revealed the diagnosis of $\mathrm{KD}$ and she was treated with IVIG ( $2 \mathrm{gr} / \mathrm{kg}$ ) and aspirin. The fever subsided within a day but restarted after 6 days. A second course of IVIG was administered. On day 4 after second course of IVIG, laboratory evaluation revealed hemoglobine level of $8.3 \mathrm{gr} / \mathrm{dL}$. Her red blood cells became positive on polyclonal IgG Coomb's testing (DAT). Aspirin was stopped and steroid was started. Her original signs had resolved and hemoglobine level gradually increased up to $11.7 \mathrm{gr} / \mathrm{dL}$. Eight months later, she readmitted with significantly enlarged servical lymph nodes in parallel with previuos symptoms consistent with the diagnosis of recurrent $\mathrm{KD}$. Hemoglobin level was $11 \mathrm{gr} / \mathrm{dL}$ and DAT was negative. Following single dose of IVIG treatment, hemoglobine level gradually decreased and became $6.6 \mathrm{gr} / \mathrm{dL}$ on 30 th day of treatment with DAT positivity. Within first week of steroid treatment, hemoglobine level incresaed to $8.9 \mathrm{gr} / \mathrm{dL}$. The patient is now free of any symptom with an hemoglobine level around $11.5 \mathrm{gr} / \mathrm{dL}$.

Conclusion It is important that physicians using high dose IVIG are aware of the risk of hemolysis. Careful monitoring of hemoglobin levels during IVIG treatment may provide proper diagnosis and early intervention.

\section{CLASSIC KAPOSI SARCOMA WITH PULMONARY INVOLVEMENT MIMICKING ENDOBRONCHIAL TUBERCULOSIS IN A CHILD}

doi:10.1136/archdischild-2012-302724.0519

${ }^{1} \mathrm{FB}$ Çakır, ${ }^{2} \mathrm{E}$ Çakır, ${ }^{3} \mathrm{E}$ Torun, ${ }^{4} \mathrm{~N}$ Tuzuner, ${ }^{5} \mathrm{~A}$ Kut. ' $D$ epartment of Pediatric Hematology; ${ }^{2}$ Department of Pediatric Pulmonology; ${ }^{3}$ Department of Pediatrics, Bezmialem Vakif University; ${ }^{4}$ Department of Pathology, Istanbul University Cerrahpasa Medical Faculty; ${ }^{5}$ Department of Pediatric Pulmonology, Sureyyapasa Chest Diseases and Thoracic Surgery Education and Research Hospital, Istanbul, Turkey

Kaposi' sarcoma (KS) is a low-grade vascular neoplasm and classic $\mathrm{KS}$, a subtype of KS, is extremely rare in children. Childhood pulmonary involvement in classic KS has not been reported in the literature. We describe an HIV-seronegative pediatric case with a fulminant course of classic KS with pulmonary involvement mimicking endobronchial tuberculosis.

\section{DIAGNOSIS AND TREATMENT PECULIARITIES IN AN} INFANT WITH BLEEDING DISORDER

doi:10.1136/archdischild-2012-302724.0520
${ }^{1} \mathrm{SI}$ lurian, ${ }^{1} \mathrm{ML}$ Neamtu, ${ }^{2} \mathrm{G}$ Gradinariu, ${ }^{3} \mathrm{~S}$ lurian, ${ }^{2} \mathrm{~A}$ Vidrighin, ${ }^{2} \mathrm{E}$ Vina. ${ }^{1}$ Research Department, Pediatric Clinic, Lucian Blaga University; ${ }^{2}$ Pediatric Clinic; ${ }^{3}$ Clinical Laboratory, Pediatric Hospital, Sibiu, Romania

Background and Aims One of bleeding causes due to vitamin $\mathrm{K}$ deficiency is gut flora destruction secondary to antibiotic treatment early in life. Authors emphasize diagnosis and treatment difficulties for an infant with massive uncontrolled bleeding.

Methods Authors present a 5 weeks-old breastfed infant transferred in pediatric clinic for severe anaemia. Family history: healthy parents, no consanguinity. Case history: recent respiratory infection treated with antibiotics; no recent trauma or surgery. Clinical exam: skin pallor, petechiae, ecchymoses, jaundice, huge haematoma $(20 / 14 \mathrm{~cm})$, wide-spread from neck to lumbar area.

Results Blood investigations: severe anaemia $(\mathrm{Hb}=3.5 \mathrm{~g} / \mathrm{dl})$, severe hyponatremia, normal liver function, negative serology for celiac disease. Negative test for cystic fibrosis. Hemostasis evaluation: normal values for bleeding time, platelets and fibrinogen; significant prolongation for prothrombin time and activated partial thromboplastin time.

Evolution: Infant developed fulminant seizures secondary to hyponatremia and bleeding at venous puncture sites, justifying urgent initiation of anticonvulsant therapy and recombinant human coagulation factor VII, even before first hemostasis evaluation. Despite of therapy, bleedings symptoms persisted and became more severe. According to hemostasis investigations, we diagnosed vitamin $\mathrm{K}$ deficiency and we reconsidered the treatment using $\mathrm{K}$ vitamin. Prompt improvement of bleeding after vitamin K therapy confirmed vitamin $\mathrm{K}$ deficiency. After blood transfusion authors noticed haemoglobin(Hgb) improvement (at discharge $\mathrm{Hgb}=14.1 \mathrm{~g} / \mathrm{dl}$ ).

\section{Conclusions}

1. Authors emphasize diagnosis and treatment difficulties in an infant with severe bleeding because of vitamin $\mathrm{K}$ deficit;

2. In cases with severe bleeding, it's mandatory to consider vitamin K treatment;

3. Antibiotic treatment should be carefully considered in infants.

\section{UNUSUAL PRESENTATION OF DISSEMINATED PANDEMIC INFLUENZA A (H1N1) 2009 IN AN INFANT}

doi:10.1136/archdischild-2012-302724.0521

'P Suandork, 'D Aranwutikul, 'S Chaisuparassameekul, ${ }^{2} \mathrm{~A}$ Thitithanyanont, ${ }^{2} \mathrm{~S}$ Wiboon-ut, ${ }^{2} \mathrm{P}$ Kanrai, ${ }^{3} \mathrm{~S}$ Worapongpaiboon, ${ }^{4} \mathrm{~S}$ Hongeng. 'Department of Pediatrics, Bangkok Hospital, Bangkok Hospital Group; 'Department of Microbiology, Faculty of Science, Mahidol University; ${ }^{3}$ Department of Pathology, Samitivej Hospital; ${ }^{4}$ Department of Pediatrics, Ramathibodi Hospital, Mahidol University, Bangkok, Thailand

Background Children are the risk group for severe disease of Pandemic influenza A (H1N1) 2009 infection. A case of disseminated manifestation of pandemic H1N1 influenza has been rarely reported. Methods We present a case of a-3-month-old male infant who manifested with clinical sepsis and can be demonstrated the evidence of disseminated pandemic H1N1 influenza in bone marrow prior having respiratory symptoms.

Results The patient presented with high fever for 1 day. The initial diagnosis was sepsis but he had persisted fever with hepatosplenomegaly. Complete blood count persistently showed pancytopenia. Bone marrow aspiration and biopsy on day 8 showed predominant population of maturing myeloid precursors. In contrast, erythroid precursors were virtually absent. PCR tested in serum was negative for Epstein-Barr virus, cytomegalovirus, dengue virus and parvovirus. On day 11, he developed respiratory distress and required ventilator support. Bronchoalveolar lavage was positive for pandemic H1N1 influenza by both RT-PCR and viral culture. The staining marrow specimens performed on day 8 with immunofluorescence 